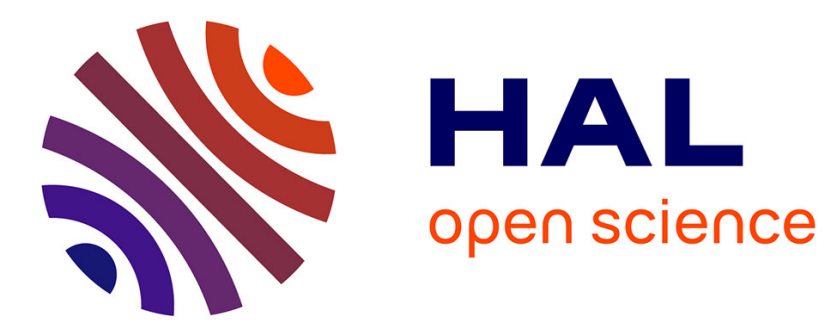

\title{
Measuring and Modeling the Growth Dynamics of Self-Catalyzed GaP Nanowire Arrays
}

Fabrice Oehler, Andrea Cattoni, Andrea Scaccabarozzi, Gilles Patriarche, Frank Glas, Jean-Christophe Harmand

\section{- To cite this version:}

Fabrice Oehler, Andrea Cattoni, Andrea Scaccabarozzi, Gilles Patriarche, Frank Glas, et al.. Measuring and Modeling the Growth Dynamics of Self-Catalyzed GaP Nanowire Arrays. Nano Letters, 2018, 18 (2), pp.701-708. 10.1021/acs.nanolett.7b03695 . hal-02413855

\section{HAL Id: hal-02413855 \\ https://hal.science/hal-02413855}

Submitted on 16 Dec 2019

HAL is a multi-disciplinary open access archive for the deposit and dissemination of scientific research documents, whether they are published or not. The documents may come from teaching and research institutions in France or abroad, or from public or private research centers.
L'archive ouverte pluridisciplinaire HAL, est destinée au dépôt et à la diffusion de documents scientifiques de niveau recherche, publiés ou non, émanant des établissements d'enseignement et de recherche français ou étrangers, des laboratoires publics ou privés. 


\section{Measuring and modeling the growth dynamics of self-catalyzed GaP nanowire arrays}

Fabrice Oehler, Andrea Cattoni, Andrea Scaccabarozzi, Gilles Patriarche, F. Glas, and Jean-christophe Harmand

Nano Lett., Just Accepted Manuscript • DOI: 10.1021/acs.nanolett.7b03695 • Publication Date (Web): 19 Dec 2017

Downloaded from http://pubs.acs.org on December 20, 2017

\section{Just Accepted}

"Just Accepted" manuscripts have been peer-reviewed and accepted for publication. They are posted online prior to technical editing, formatting for publication and author proofing. The American Chemical Society provides "Just Accepted" as a free service to the research community to expedite the dissemination of scientific material as soon as possible after acceptance. "Just Accepted" manuscripts appear in full in PDF format accompanied by an HTML abstract. "Just Accepted" manuscripts have been fully peer reviewed, but should not be considered the official version of record. They are accessible to all readers and citable by the Digital Object Identifier (DOI®). "Just Accepted" is an optional service offered to authors. Therefore, the "Just Accepted" Web site may not include all articles that will be published in the journal. After a manuscript is technically edited and formatted, it will be removed from the "Just Accepted" Web site and published as an ASAP article. Note that technical editing may introduce minor changes to the manuscript text and/or graphics which could affect content, and all legal disclaimers and ethical guidelines that apply to the journal pertain. ACS cannot be held responsible for errors or consequences arising from the use of information contained in these "Just Accepted" manuscripts. 


\title{
Measuring and modeling the growth dynamics of self-catalyzed GaP nanowire arrays
}

\author{
Fabrice Oehler, ${ }^{*, \dagger}$ Andrea Cattoni, ${ }^{\dagger}$ Andrea Scaccabarozzi, ${ }^{\dagger}, \stackrel{\dagger}{\dagger}$ Gilles Patriarche, ${ }^{\dagger}$ \\ Frank Glas, $^{\dagger}$ and Jean-Christophe Harmand ${ }^{\dagger}$ \\ $\dagger$ †entre for Nanoscience and Nanotechnology, CNRS, Université Paris-Sud, Université \\ Paris-Saclay, Route de Nozay, 91460 Marcoussis, France \\ $\ddagger$ Institut Photovoltä̈que d'Ile-de-France, 92160 Antony, France \\ E-mail: fabrice.oehler@c2n.upsaclay.fr
}

\begin{abstract}
The bottom-up fabrication of regular nanowires (NWs) arrays on a masked substrate is technologically relevant but the growth dynamic is rather complex due to the superposition of severe shadowing effects that vary with array pitch, NW diameter, NW height and growth duration. By inserting GaAsP marker layers at a regular time interval during the growth of a self-catalyzed GaP NW array, we are able to retrieve precisely the time evolution of the diameter and height of a single NW. We then propose a simple numerical scheme which fully computes shadowing effects at play in infinite arrays of NWs. By confronting the simulated and experimental results, we infer that re-emission of Ga from the mask is necessary to sustain the NW growth while Ga migration on the mask must be negligible. When compared to random cosine or random uniform re-emission from the mask, the simple case of specular reflection on the mask gives the most accurate account of the Ga balance during the growth.
\end{abstract}




\section{Keywords}

GaP nanowires, self-catalyzed, molecular beam epitaxy, nanowire arrays, axial growth, radial growth, shadowing, numerical simulation

\section{Introduction}

Nanowires (NWs) grown using the Vapour-Liquid-Solid (VLS) mechanism have attracted much attention. This is due in part to their interesting growth physics and to the variety of geometries that can be obtained. Alternatively to standard VLS growth assisted by a foreign metal catalyst (such as Au), some semiconducting III-V NWs can grow with the VLS droplet material overwhelmingly consisting of the group III metal. ${ }^{1-3}$ This 'self-catalyzed' VLS mode, which has been realized mainly in molecular beam epitaxy (MBE), allows one to modify the droplet volume during growth by changing the ratio of the fluxes of group III and group V species. ${ }^{4,5}$ Recently, modeling and experiments have indeed shown the effect of this ratio on the NW diameter. In particular, a mechanism driving ensemble of nanowires toward a single stable geometry (height, diameter) has been found, depending directly on the material flux and growth conditions. ${ }^{6,7}$

The iconic material system for such self-catalyzed VLS growth is Ga-catalyzed GaAs NWs. In GaAs NWs, the contact angle appears to dictate the selection between cubic zinc blende and hexagonal wurtzite. ${ }^{5}$ For other semiconductors that have an indirect band gap in the cubic phase, such as GaP, the opportunity to obtain the hexagonal structure with direct band gap may be of importance. ${ }^{8-10}$

The self-catalyzed MBE growth of GaAs and GaP NWs occurs under very similar growth conditions (substrate temperature, group III and group V fluxes), so that ternary Ga(As,P) NWs can be grown within the full compositional range simply by altering the composition of the group V flux (P/As ratio). ${ }^{11,12}$ Besides possible applications, the growth compatibility between different materials is useful for fabricating thin marker layers which may be inserted 
at regular time intervals to monitor the growth dynamics, as done for $\operatorname{InAs}(\mathrm{P}),{ }^{13} \operatorname{In}(\mathrm{As}, \mathrm{P}){ }^{14}$ or $\mathrm{Ga}(\mathrm{Al}) \mathrm{As}^{15} \mathrm{NWs}$. In the particular case of $\mathrm{GaP}, \mathrm{Ga}(\mathrm{As}, \mathrm{P})$ markers have been used to characterize the kinetics of self-catalyzed ${ }^{12}$ and Au-catalyzed ${ }^{16}$ NWs.

Recent work on $\mathrm{GaP}^{17}$ and $\mathrm{GaAs}^{18} \mathrm{NW}$ arrays grown by MBE have demonstrated the importance of local V:III ratios on the NW morphology and growth dynamics. However the direct in situ observation of the effects of specific growth conditions in such NW arrays is complex. Previous attempts using in situ XRD could only resolve some features of NW growth, ${ }^{19}$ but more recent studies at synchrotron facilities now combines ex situ scanning electron microscopy (SEM) with time-resolved in situ X-ray diffraction (XRD) to start sorting out the axial and radial growth dynamics of single self-catalyzed GaAs NWs. ${ }^{20}$ Several authors have attempted to model the growth dynamics of NW arrays, yet with several approximations. Dalacu et al. ${ }^{21}$ propose a model in which NWs grow from a direct flux and a reflected flux originating from the mask but no shadowing is taken into consideration. Kelrich et al. ${ }^{22}$ propose a 'line-of-sight' model for shadowing of the reflected flux, but the direct flux is not shadowed. Gibson et al. ${ }^{23}$ consider shadowing for both the group III and group $\mathrm{V}$ fluxes but several approximations are used. These works ${ }^{21-23}$ also presume that the re-emitted flux from the mask follows a cosine law (i.e. Lambertian scattering) without physical justification. Alternatively, Madsen et al. ${ }^{13}$ do not consider a re-emitted flux but a diffusion flux on the surface around each NW, accounting for shadowing effects. The stochastic numerical method developed by Sabelfeld et al. ${ }^{24,25}$ shares some starting hypotheses with the present model but deals about randomly positioned NWs of different height while we focus on uniform NWs growing in regular arrays.

The present work combines and improves on these previous experimental and modelling efforts. We measure here the evolution of axial and radial growth rates with unprecedented time resolution. By effectively disentangling axial and radial growth and performing detailed simulations of the impinging fluxes, we are able to discriminate between the different material pathways such as direct impingement, surface migration or evaporation from the mask. The 
fundamental understanding of growth processes that ensues is critical for the successful fabrication of devices based on bottom-up NW technology.

\section{Results and discussion}

The self-catalyzed GaP NWs were grown by MBE on a (111) Si substrate. The NW growth kinetics are monitored by the insertion of $122 \mathrm{Ga}(\mathrm{As}, \mathrm{P})$ markers at fixed time intervals $\delta t=30 \mathrm{~s}$, so that the total growth time is about $1 \mathrm{~h}$. More details about the growth procedure can be found in the experimental section. Figure 1.a shows a typical SEM bird's eye view of the hexagonal array (500 nm pitch) of GaP NWs. Excluding the very edge of the pattern, the NW morphology is uniform with average diameter and height of respectively $140 \mathrm{~nm}$ and $3.2 \mu \mathrm{m}$. The yield of vertical NWs is $80-85 \%$ over the whole array. Figure 1.b shows a top view image in which the red dotted line represents the slice extracted using a focused ion beam (FIB) for cross-sectional analysis by transmission electron microscopy (TEM). Figure 1.c shows the corresponding bright field TEM image, with a row of vertical GaP NWs on the (111) Si substrate. A top layer of platinum has been deposited to protect the NWs from FIB damage. Areas of interest along the NW are identified by black rectangles labeled by letters (d-g) and these zones are further investigated in Fig. 1.d-1.g using scanning TEM high angle annular dark field (HAADF) imaging to obtain chemical contrast. HAADF images (Fig. 1.d-1.g) clearly reveal the Ga(As,P) markers which appear brighter than the GaP matrix due to a higher average atomic number. The Ga(As,P) marker thickness is nearly constant in the axial direction $(5-6 \mathrm{~nm})$, but the radial extension of the marker increases continuously with marker index: it is $\sim 60 \mathrm{~nm}$ at marker \#31 (Fig. 1.g) and very close to the final wire diameter $(\sim 144 \mathrm{~nm})$ near marker \#106 (Fig. 1.d). The radial extension of the GaAsP marker is indicated by a dotted white line in Fig. 1.d-g. Small wire to wire variations exist but their amplitude is always smaller than the difference between two consecutive markers in a single wire (see Supplementary Information S1). 

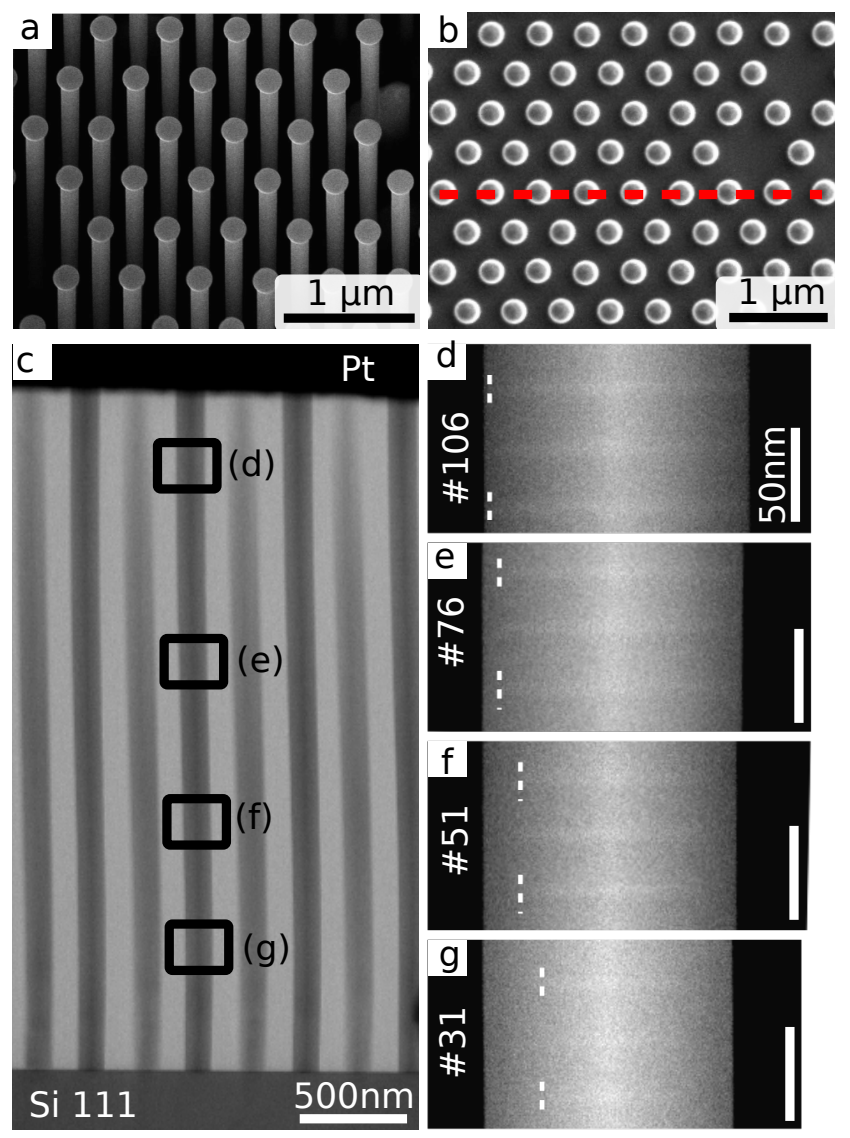

Figure 1: (a) SEM bird's-eye view of the hexagonal NW array. (b) Top-view SEM image with position of the FIB cut marked by dashed red line. (c) Medium resolution crosssectional TEM image showing vertical GaP NWs on the (111) Si substrate. Black rectangles mark the zones further analysed by HAADF imaging. (d)-(g) Zoomed in HAADF scanning TEM images showing the Ga(As,P ) markers at different positions along the GaP NW axis (indicated by marker index). Labels (d)-(g) match those of the corresponding rectangles in (c). Dotted white lines indicate the radial extension of neighbouring $\mathrm{Ga}(\mathrm{As}, \mathrm{P})$ markers.

In Figure 2.a we report the time evolution of the instantaneous axial growth rate of the NW together with the beam equivalent pressure (BEP) of phosphorus evaluated during growth. More details about BEP measurement can be found in Supplementary Information S2. The axial growth rate is computed from the distance between two consecutive markers. We observe two distinct regimes: from marker \#4 to \#26 (2-13 min), the axial growth rate steadily increases from 0.3 to $1 \mathrm{~nm} \mathrm{~s}^{-1}$; it then remains nearly constant from marker \#26 to \#120 (13-60 min). We observe that the $\mathrm{P}$ source suffered from two bursts, i.e. sharp increases in the group V BEP, leading to increased axial growth rates at markers \#22-23 
and $\# 75$.
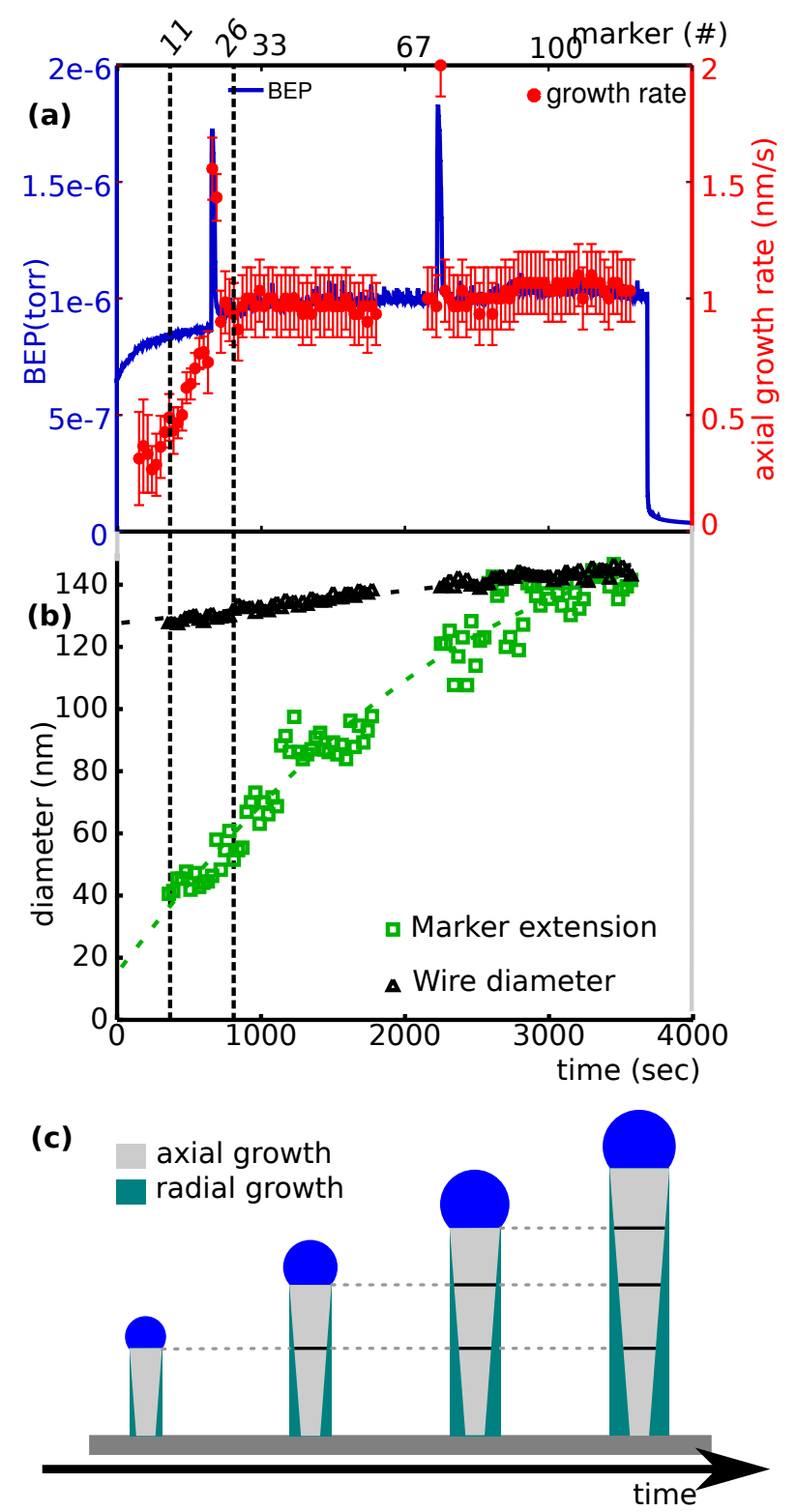

Figure 2: Evolution of the NW geometry and phosphorus pressure (BEP) with time or marker index (a marker is inserted every 30s). (a) Evolution of the $\mathrm{P}_{2} \mathrm{BEP}$ and $\mathrm{NW}$ axial growth rate with time. The missing data between markers \#60 and 71 is due to a missing TEM image at sufficiently high resolution. (b) Measurement of the radial extension of the $\mathrm{Ga}(\mathrm{As}, \mathrm{P})$ markers (green squares) and of the final wire diameter at the same position (black triangles). Marker \#26 (13 min growth) marks the border between regimes of increasing and constant axial growth rates. The radial extension of the markers could not be measured below marker \#11. (c) Schematics of the evolution of NW morphology resulting from simultaneous axial and radial growth. Example marker layers are indicated by black lines. 
Figure 2.b shows the radial extension of the markers and the final diameter of the NW measured at each marker position. We observe a minute reverse tapering of the final NW (about $0.1^{\circ}$, corresponding to a diameter variation from $129 \mathrm{~nm}$ to $144 \mathrm{~nm}$ over $3.2 \mu \mathrm{m}$ length). In contrast, the radial extension of the $\mathrm{Ga}(\mathrm{As}, \mathrm{P})$ markers changes by a factor of 3 over the total growth duration, from about $40 \mathrm{~nm}$ at marker \#11 to over $140 \mathrm{~nm}$ from marker \#90 onwards. It is important to acquire images along the $[11 \overline{2}]$ zone axis, parallel to the $[1 \overline{1} 0]$ facets of the NW, to avoid projection issues when determining the radial extension of the marker. Still, the chemical contrast between $\mathrm{Ga}(\mathrm{As}, \mathrm{P})$ marker and GaP matrix is limited and the dispersion of the marker radial extension (green squares) is large compared to that in the final wire diameter (black triangles), for which the edge of the NW is clearly defined. A typical measurement of the radial extension of a GaAsP marker is shown in Supplementary Information S3.

The axial position of the early markers (\#4-10) could only be determined using integrated axial profiles and no radial extension could be measured. The positions of very first markers \#1-3 could not be unambiguously determined and are excluded from the analysis. This is a direct consequence of the experimental design, which uses a small As content in the marker to avoid disturbing the GaP NW growth. Quantitative chemical analysis (see supplementary information S4) indicates that the nominally pure GaP matrix is actually a ternary $\mathrm{GaAs}_{\mathrm{x}} \mathrm{P}_{1-\mathrm{x}}$ alloy with $x=0.02$, while the composition of the markers is typically $x=0.06$. This incorporation of As in the nominally pure GaP sections of the NW is most likely a consequence of the residual $\mathrm{As}_{4}$ background when the As source is closed.

In previous growth experiments on self-catalysed GaAs NW using (Al,Ga)As or Ga(As,P) markers, it is typically observed that the radial extension of the marker matches the diameter of the wire in the absence of tapering. ${ }^{12,26}$ Moreover the small difference in alloy composition between matrix and markers, corresponding to $\delta x=0.04$, are unlikely to create enough strain to change that situation by favouring island growth. ${ }^{27}$ It is thus reasonable to consider that the radial extension of the marker is a snapshot of the NW diameter when the marker was 
grown.

At the end of growth, the NW presents nearly vertical sidewalls. As no change in the growth condition was deliberately introduced in the experiment, we may assume that the resulting quasi-cylindrical shape also pertains to the earlier stages of growth, at least in the constant axial growth rate regime (\#26-122). The corresponding evolution of NW morphology with time is illustrated schematically in Fig. 2.c, highlighting the contributions from axial and radial growth.

These results constitute the first instance of simultaneous measurements of diameter and axial growth rate variations in a single NW (GaP), besides indirect in situ XRD measurements on GaAs using a synchrotron. ${ }^{20}$ Moreover, our data cover an extended period of time $(\sim 1 \mathrm{~h})$ and our MBE machine operates in standard growth conditions as regards substrate heating and rotation and solid source cells. In particular, the steady state value of the axial growth rate, $1 \mathrm{~nm} . \mathrm{s}^{-1}$, is similar to what has been reported in self-catalyzed GaAs NW growth studies. ${ }^{2,15,28}$ Yet, we find a peculiar situation in which the GaP NWs are not tapered whereas their instantaneous diameter varies significantly during growth. Again, this has been previously observed in GaAs NWs arrays ${ }^{23}$ but recent theoretical studies do not consider this phenomenon. ${ }^{6,7}$

A particular aspect of growth in regular arrays is the upper limit imposed on the material input to each NW. For isolated or low density NWs, the total amount of material reaching the NW per unit time (hereafter termed current) can be arbitrarily large due to diffusion from the substrate, re-emission from the substrate and direct capture by the sidewalls, possibly followed by surface diffusion. On the other hand, the growth of a NW in a regular array is limited by the amount of material hitting the primitive unit cell surrounding each NW. For an hexagonal array of pitch $p$, the unit cell area is $p^{2} \sqrt{3} / 2$. When exposed to a Ga flux $\phi_{2 d}$ (measured per unit area of the substrate), the maximum Ga current $I_{m}$ available for growth is simply given by: 
2

3

4

5

6

7

8

10
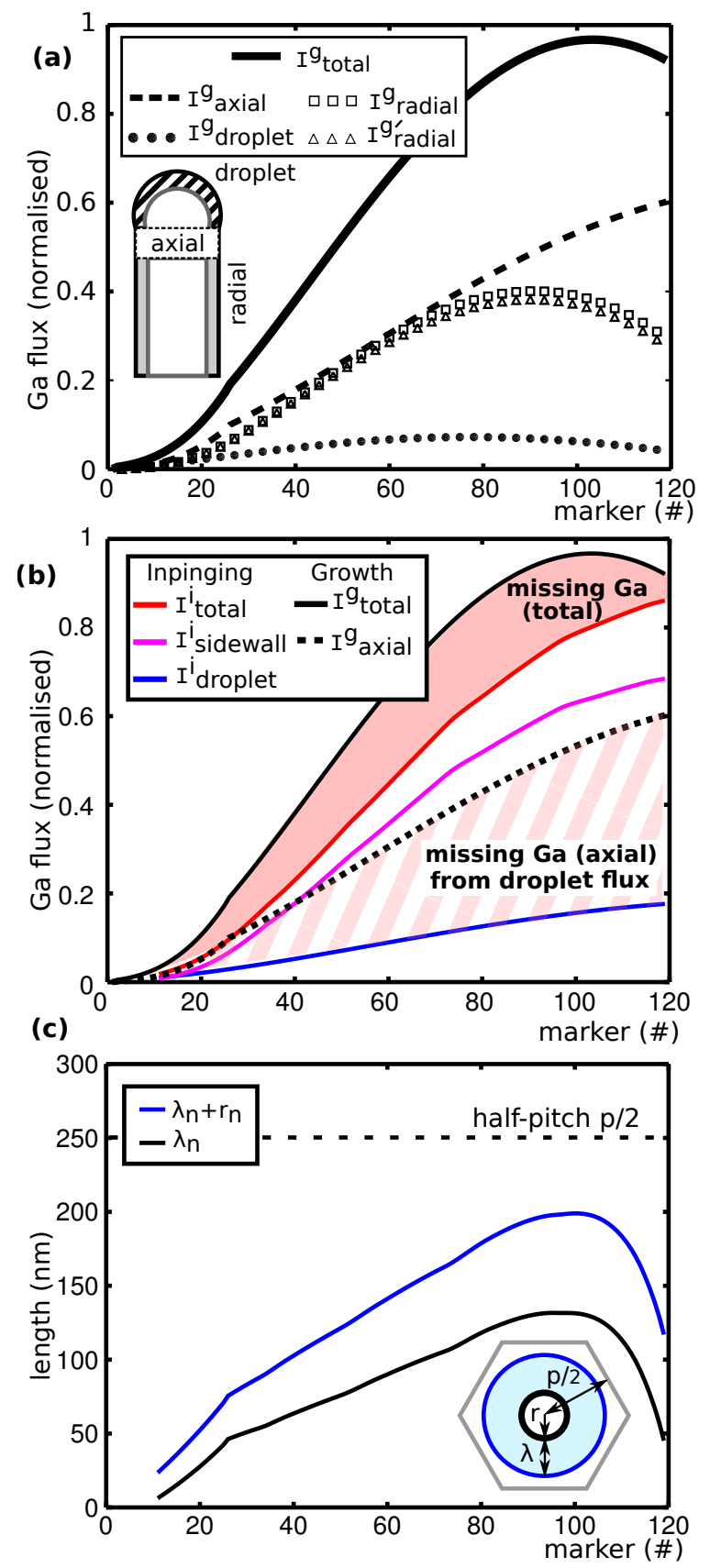

Figure 3: Evolution of the measured and calculated Ga currents with time (indicated by marker index). (a) Ga currents consumed by axial, radial growth and droplet enlargement normalized to the Ga impinging on the unit cell evaluated from the position and width of the marker layers. (b) Simulated Ga currents directly impinging on the NW sidewalls and droplet (colored curves) compared to measured Ga current consumed by the growth (black curves). Pink striped and solid areas represent the missing Ga. (c) Time evolution of collection length $\lambda$ necessary to account for missing Ga if only Ga diffusion on mask is considered. 


$$
I_{m}=\frac{p^{2} \sqrt{3}}{2} \phi_{2 d}
$$

Flux $\phi_{2 d}$ is easily obtained from the two-dimensional (2D) growth rate measured on a planar (001) GaAs surface, namely here $0.135 \mathrm{~nm} \mathrm{~s}^{-1}$, which converts into $\phi_{2 d} \simeq 3.00 \mathrm{at} \mathrm{nm}^{-2} \mathrm{~s}^{-1}$.

From the measured marker height positions $h_{n}$ and radii $r_{n}$ (Fig. 2), we can compute the instantaneous radial and axial growth rates and the radial and axial volumes of material created between each pair of markers (see insert, Fig. 3.a). From marker $n$ to $n+1$, the radius and height of the NW increase so that the new Ga material distributes between three contributions, namely the volumes $\Delta V_{\text {axial }}$ and $\Delta V_{\text {radial }}$ created by axial and radial growth, respectively, and the change of droplet volume $\Delta V_{\text {droplet }}$ :

$$
\begin{aligned}
\Delta V_{\text {axial }} & =\pi r_{n+1}^{2}\left(h_{n+1}-h_{n}\right) \\
\Delta V_{\text {radial }} & =\pi h_{n}\left(r_{n+1}^{2}-r_{n}^{2}\right) \\
\Delta V_{\text {droplet }} & =\pi\left(r_{n+1}^{3}-r_{n}^{3}\right) \frac{(1-\cos \beta)^{2}(2+\cos \beta)}{3 \sin ^{3} \beta}
\end{aligned}
$$

where the third equation is obtained assuming a constant contact angle $\beta=135^{\circ}$. Eq. (2) allows one to assess directly the extra volumes created by axial and radial growth and by droplet enlargement from the axial positions and widths of the markers. The expression for $\Delta V_{\text {radial }}$ in Eq. (2) can be modified to account for NWs with a uniform tapering angle $\alpha$, counted positive (from the vertical) for reverse tapering:

$$
\Delta V_{\text {radial }}^{\prime}=\pi h_{n}\left[\left(r_{n+1}^{2}-r_{n}^{2}\right)-h_{n}\left(r_{n+1}-r_{n}\right) \tan \alpha\right]
$$

The instantaneous currents of Ga atoms $I_{\text {axial }}^{g}, I_{\text {radial }}^{g}$ and $I_{\text {droplet }}^{g}$ are then computed by converting the volume changes over time $\delta t$ given by Eq. (2) using the appropriate elementary volume $\omega$ (atomic pair for the solid $\omega_{G a P}=0.04047 \mathrm{~nm}^{3}$, Ga atom for the liquid $\omega_{G a(l)}=$ $\left.0.02027 \mathrm{~nm}^{3}\right)$ : 


$$
I_{i}^{g}=\frac{\Delta V_{i}}{\delta t \omega}
$$

The total rate of consumption of Ga (accounting for the small wire tapering) is :

$$
I_{\text {total }}^{g}=I_{\text {droplet }}^{g}+I_{\text {axial }}^{g}+I_{\text {radial }}^{\prime g}
$$

In the following discussion, the currents will be normalized to the maximum current $I_{m}$ per unit cell of the array, given by eq.(1).

Fig. 3.a shows the values of the three normalized currents $I^{g}$ at each marker position calculated using Eqs.(2-4). To obtain smoother curves, we did not use the raw measurements of $r_{n}$ and $h_{n}$ but a parabolic fit of the marker extension (Fig. 2.b, dotted green line) and two fits of the axial growth rate (linear between markers \# 1 and 26 and constant thereafter; see Fig. 2.a). We observe that the axial and radial currents $I_{\text {axial }}^{g}$ and $I_{\text {radial }}^{g}$ are comparable during the whole experiment whereas the amount of Ga used for droplet enlargement $I_{\text {droplet }}^{g}$ is very minor. As expected from the small value of the reverse tapering angle $\left(\alpha \simeq 0.1^{\circ}\right)$, the current $I_{\text {radial }}^{\prime g}$ obtained accounting for reverse tapering is only marginally inferior to the vertical sidewall approximation $I_{\text {radial }}^{g}$. In our growth conditions, the Ga material used thus splits nearly evenly between axial and radial incorporation for most of the growth duration. The total amount of Ga used for NW growth (and droplet inflation) is initially small compared to $I_{m}$ whereas it nearly reaches the physical limit $I_{\text {total }}^{g} \simeq I_{m}$ toward the end of growth. This demonstrates that NWs in an array may be very efficient (at better than $90 \%$ ) at collecting not only the direct flux hitting the NW surface but all the Ga material that enters the associated unit cell of the array.

The final drop in the total Ga consumption $I_{\text {total }}^{g}$ from marker \#110 onward may be an artefact related to the incorrect evaluation of $I_{\text {radial }}^{g}$. At this point the wire is very long, over $3 \mu \mathrm{m}$, and a small enlargement of the radius corresponds to the crystallisation of very large volume $\mathrm{GaP}$ due to radial growth $I_{\text {radial }}^{g}$. It is very likely that the simple parabolic fit of 
the radius does not capture such fine details, resulting in a reduced accuracy in the growth currents at the final stages of the growth between markers \#110-120.

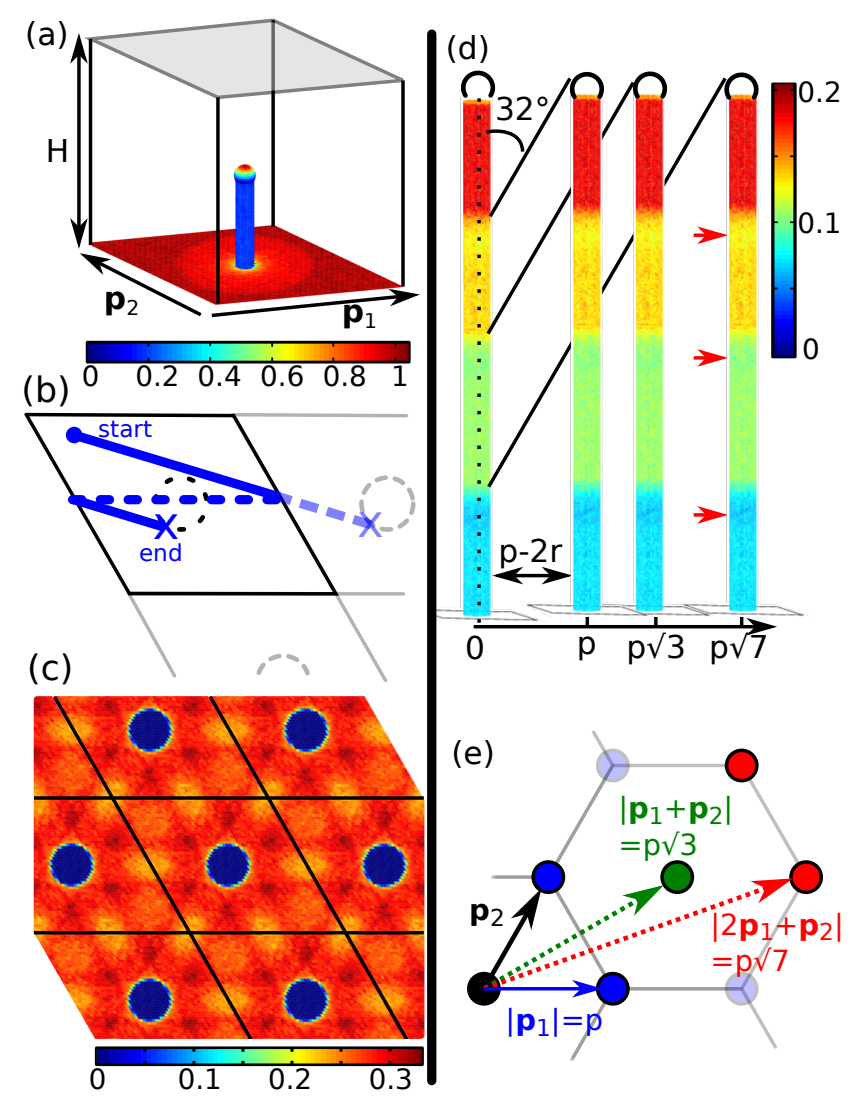

Figure 4: Simulation of the impinging Ga flux. All colorbars are normalized so that unity is the reference 2D-growth hit density. (a) Schematics of the rhombic unit cell used for the numerical computations. Here the short NW only shadows the surrounding surface of the mask but not its neighbors. (b) Illustration of the periodic boundary conditions used to simulate the ballistic trajectories. (c) Example of hit pattern resulting from the complex shadowing of the mask by several NWs. The unit-cell is replicated to better show the hexagonal symmetry. (d) Illustration of the shadowing of the NW sidewalls by several neighbor NWs located at different distances. The incident Ga beam is inclined at $32^{\circ}$ from the substrate normal. Red arrows mark the deeper shadows caused by the droplets with contact angle $\beta=135^{\circ}$. (e) Schematics of an hexagonal array showing the positions of the first shadowing neighbors and the corresponding distances.

We now compare this experimental determination of the various rates of consumption of $\mathrm{Ga}\left(I^{g}\right)$ to simulations carried out considering increasingly diverse material pathways. Since the NWs are organized in a regular hexagonal array, the material flux impinging on each NW can be fully determined. However, the calculation is made difficult by shadowing 
of the bottom $\mathrm{SiO}_{2}$ surface (mask) by the NWs and between the NWs themselves. We propose a simple numerical scheme which allows one to simulate an infinite hexagonal array (Figure 4). We first define an hexagonal unit cell that contains a single wire at its center; the base of the cell is a rhombus with base vectors $\mathbf{p}_{1}$ and $\mathbf{p}_{2}$ at a $60^{\circ}$ angle and its height $H$ is arbitrary (Fig. 4.a). This rhombus-based unit cell has a simpler shape than the typical hexagon so that the computation is simpler, but both unit cells have the same area (this shape can also easily be adapted to simulate other kinds of infinite arrays, such as square or rectangular). Provided the NW height is smaller than $H$, we can quickly simulate an infinite hexagonal array of identical NWs by applying periodic boundary conditions to a single rhombic cell. To simplify the calculations, we approximate the NW by a circular cylinder and the catalyst droplet by a spherical cap. We model the external flux as particles with random and uniformly distributed entry points on the top surface of the unit cell (Fig. 4.a, light gray). Each particle enters the cell at a given angle from the substrate normal (here $32^{\circ}$ to account for the elevation of the Ga MBE cell) but with a random in-plane orientation to simulate substrate rotation. The trajectory of each particle is then computed as follows. The particle propagates in a series of straight segments. If it hits a vertical cell boundary, it is simply translated by the appropriate base vector to reenter the unit cell with the same trajectory direction (Fig. 4.b, blue line). As regards shadowing and re-emission from the mask, we will consider a series of hypotheses.

To verify the validity of our simulation scheme, we first consider that the particle terminates when it hits either the NW sidewall, or the catalyst surface or else the floor of the cell (substrate or $\mathrm{SiO}_{2}$ mask). This is equivalent to considering the flux directly impinging on the nanowire or the mask surface. For short and thin NWs, the NWs shadow the neighboring surface but there is no mutual shadowing between NWs. This pertains to short and thin NWs or large pitches. Figure 4.a shows the result of such a simulation (pitch $\mathrm{p}=500 \mathrm{~nm}$, NW radius $\mathrm{r}=25 \mathrm{~nm}, \mathrm{NW}$ height $\mathrm{h}=242 \mathrm{~nm}$, droplet contact angle $\beta=135^{\circ}$ ) corresponding to the NW geometry at marker \#10. In this simulation, $10^{7}$ different particle trajectories 
were used to compute the hit density. The rotational symmetry of the latter indicates that our simulation scheme accounts correctly for substrate rotation.

With taller NWs (assumed to have a uniform size at each instant), the same surface patch will be shadowed differently by several NWs. The hit density on the mask now exhibits a complex pattern, such as shown in Fig. 4.c $\left(\mathrm{p}=500 \mathrm{~nm}, \mathrm{r}=72 \mathrm{~nm}, \mathrm{~h}=3240 \mathrm{~nm}, \beta=135^{\circ}\right)$ corresponding to marker \#90. Because it depends on the NW neighbours, represented here by the periodic boundary conditions, the computed hit density on the cell floor respects the hexagonal symmetry of the array. In addition to the Ga densities on the mask, we also obtain the shadowing of the NW sidewalls caused by the neighbouring wires. Figure. 4.d shows the corresponding hit density. The image of the central NW has been replicated at different distances $(0, p, p \sqrt{3}, p \sqrt{7})$ corresponding to the first layers of neighbors in an hexagonal array of pitch $p$ (Fig. 4.e) with a direct line of sight to the central NW. Selection of shadowing neighbours based on 'line of sight' have been previously used by Madsen et al. ${ }^{13}$ and Kelrich et al. ${ }^{22}$ As seen in Fig. 4.d, the variation of the hit density along the NW height is simply explained by the shadowing of the $32^{\circ}$-inclined flux by these successive neighbors. The small variations in the segments of near uniform hit density (Fig. 4.d, red arrows) are due to the catalyst droplet, which has a radius larger than the wire (since $\beta=135^{\circ}$ ) and thus casts a darker shadow at the top of each segment. Very importantly, the shadowing of the direct flux on the NW sidewalls changes along its height and its structure depends on the total height of the wire.

These numerical simulations reveal how the impinging Ga distributes between the droplet $\left(I_{\text {droplet }}^{i}\right)$ and the NW sidewalls $\left(I_{\text {sidewall }}^{i}\right)$ for each previously measured NW geometry (height, diameter; see Fig. 2). These currents are also normalized to the total external input in a unit cell so that we can directly compare the simulation results to the measured instantaneous atomic currents of Fig. 3.a.

In Fig. 3.b, we can now compare the measured axial and total atomic Ga growth currents with the simulated currents directly impinging on the droplet and on the sidewalls 

throughout the growth, the Ga flux hitting the droplet directly (blue curve) is clearly insufficient to provide the material used for axial growth (black dashed curve); the 'missing' Ga is represented by the pink striped area. On the other hand, there is enough Ga hitting the NW sidewalls (pink curve) to account for this missing material; we may assume, as proposed in many growth models, ${ }^{14,29,30}$ that the necessary atoms migrate to the catalyst by surface diffusion.

This analysis can be extended beyond axial growth. We may indeed compare the total amount of Ga used for axial and radial growth and droplet enlargement (Fig. 3.b, black curve) to the total amount hitting droplet and NW sidewalls, including shadowing by the neighboring wires (Fig. 3.b, red curve). It appears that these two contributions are not enough to account for the total consumption (the missing amount being represented by the uniform pink area) and this holds throughout our experiment.

However, in addition to the NW, the silica mask also collects a fraction of the direct Ga flux, which can be simply computed as $1-I_{\text {droplet }}^{i}-I_{\text {sidewall }}^{i}=1-I_{\text {total }}^{i}$. This flux is in principle sufficient to account for the 'missing' Ga (pink area), provided it can be transferred to the NW.

This transfer could occur by surface diffusion on the mask. Let us examine if this could be the case. To this end, we define the collection length $\lambda_{n}$ at the moment when marker $\# n$ is grown, as the width of the ring around the wire of radius $r_{n}$ required to provide the missing Ga material (Fig. 3.c). Specifying the dependence of the currents on $n$, we thus have:

$$
I_{\text {total }}^{g}(n)-I_{\text {total }}^{i}(n)=\frac{1-I_{\text {total }}^{i}(n)}{p^{2} \sqrt{3} / 2} \pi\left[\left(r_{n}+\lambda_{n}\right)^{2}-r_{n}^{2}\right]
$$

As shown in Fig. 3.c, this requires the collection length $\lambda_{n}$ to vary widely (from 10 to $140 \mathrm{~nm}$ ) over the growth duration. At the beginning of growth, the NWs are short and thin so they do not cast a large shadow on the mask; with a large Ga flux impinging on the mask 
and a small total Ga consumption rate $\left(I_{\text {total }}^{g} \ll 1\right)$, we compute a small value for $\lambda$. At the end of growth, the total consumption rate is $I_{\text {total }}^{g} \sim 1$, which means that nearly all the Ga impinging of the mask needs to reach the NW, so that the collection ring nearly spans the full unit cell $(\lambda \simeq p / 2-R)$. The Ga migration must be driven by the chemical potential difference of Ga between mask and NW surfaces. The variation of $\lambda$ with time would require a complex variation of the chemical potentials so that the NW could collect more and more Ga (increasing $\lambda$ ) even if the surface concentration of Ga adatoms on the mask is decreasing due to increased shadowing from the growing neighbors. It is thus unlikely that surface migration is the main mechanism for the transfer of Ga between the mask and the NW, in accordance with Krogstrup et al. ${ }^{31}$ who report very short diffusion length for Ga adatoms on $\mathrm{SiO}_{2}$ surfaces. Therefore the model of Masden et al. ${ }^{13}$ cannot be used to describe the growth of our GaP NW array.

Several authors have pointed out that, in addition to diffusion, material may be transferred from the substrate by re-emission followed by capture by the NW or the droplet. ${ }^{3,15,32}$ Again, our simulations allow us to calculate the fractions of the incident flux which are transferred in this way from the substrate to the droplet $\left(I_{d r o p l e t}^{r}\right)$ and to the NW sidewalls $\left(I_{\text {sidewall }}^{r}\right)$. Since we do not observe growth on the mask, we assume that all Ga re-evaporates from it. It is important to note that shadowing effects play an even larger role in these conditions. The direct flux is first shadowed by the surrounding NWs before it can reach the mask or the NW surface (Fig. 5.a, black lines and arrows), but the re-emitted flux is also shadowed by the neighboring NWs before it can reach the final NW (Fig. 5.a, red arrows).

The calculation of this effect requires that we specify the angular distribution of the reemitted Ga. We test three simple hypotheses. In Fig. 5.b and 5.c the angle of re-emission of each particle is respectively given by a random uniform (random re-emitter) or random cosine (Lambert re-emitter) distribution in the free half space. In both cases, we remark that the re-emitted flux hitting the droplet $I_{d r o p l e t}^{r}$ is then negligible, due to the small solid angles subtended by the droplet at the mask level. For the random uniform re-emission 
(a)
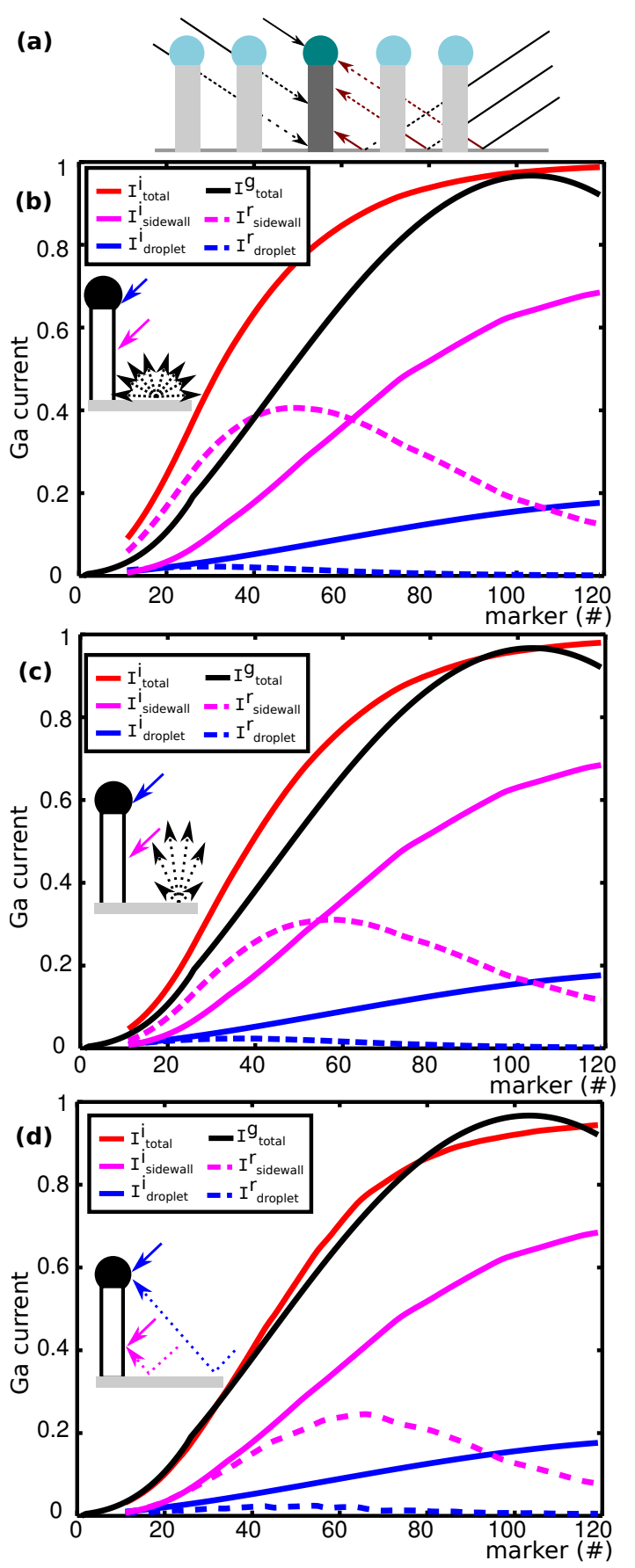

Figure 5: Comparison of measured and calculated Ga currents with re-emission of Ga from the mask. (a) Schematics of the shadowing of a selected NW by its neighbors : direct flux shadowing (dark arrows) and re-emitted flux shadowing (red arrows). (b) Gallium balance in the case of a random uniform angular distribution of the re-emitted Ga from the mask. (c) Case of a Lambert re-emitter (cosine law). (d) Case of specular reflection on the mask with $32^{\circ}$ inclination from the substrate normal. 
(Fig. 5.b) the extra flux hitting the sidewalls $I_{\text {sidewall }}^{r}$ is so large that the NW now collects an excess of Ga material, $I_{\text {total }}^{i}>I_{\text {total }}^{g}$, for the largest part of the growth duration. The random cosine re-emission (Fig. 5.c) is the typical situation considered in the literature ${ }^{13,22,23}$ due to mathematical reason. However it faces the same issue as the random uniform re-emitter, but with reduced Ga excess. Such imbalances of Ga are admissible only if we allow a fraction of the Ga atoms hitting the sidewalls to re-evaporate. The actual calculation is more complex than simply adding a scaling factor, since shadowing effects will again take place when some of the evaporating Ga atoms will hit the surrounding NWs and be unable to escape the array.

The random re-emissions considered in Fig. 5.b and 5.c are simple ways to account for the adsorption-evaporation process, during which the particle 'forgets' its initial angle of incidence. In Fig. 5.d, we consider instead the opposite case of specular reflection for the re-emission of $\mathrm{Ga}$ from the mask. As in the random re-emissions, we find that a negligible Ga transfer $I_{\text {droplet }}^{r}$ occurs from mask to droplet and a large transfer $I_{\text {sidewall }}^{r}$ from mask to sidewalls. This time, there is a good agreement between the computation of the collected Ga current $I_{\text {total }}^{i}$ (red curve) and the measured consumption of Ga $I_{\text {total }}^{g}$ (black curve). Hence the simple model of specular reflection gives a rather accurate picture of the capture of the Ga flux during most of the growth duration. This mechanism probably requires the mask to have a flat and smooth surface and the particles not to stick to it, even for a short time. This is consistent with the total absence of deposition on the mask, even far from the patterned area. Additional simulations performed with other Ga beam inclination angles show that the agreement pertains for angles within $32 \pm 2^{\circ}$ from the substrate normal.

We stress that these three models of re-emission do not involve any free parameter: the system geometry is fixed at each computation step (hexagonal array of pitch $p$, NWs of given length and diameters, as deduced from the markers) and the angle of incidence of the Ga beam is well defined $\left(32^{\circ}\right)$. There is thus no way of adjusting the results by fitting a hidden parameter: either the $\mathrm{Ga}$ is balanced between impinging atoms and the volumes consumed 
for growth and droplet enlargement or it is not. Despite its simplicity, it is remarkable that the specular reflection model gives an excellent account of the Ga distribution in a complex and evolving environment with changing NW heights, diameters and shadowing effects.

In the light of the Gallium re-emission model proposed above, it is interesting to rediscuss the experimental results presented in Fig. 2. We make here the usual hypothesis that the NW axial growth rate is limited by the group V element. ${ }^{2,3}$ As the present model only deals with Ga, we cannot explain the different regimes of axial growth rate observed experimentally (linear increase between marker \#11-26, constant value thereafter). However we can state that there is just enough Ga collected, at each step, to sustain simultaneously axial and radial growth.

Similarly, the model cannot assert how the Ga collected by the NW (through direct flux or re-emission) redistributes in the GaP volumes created by axial and radial growth. Even if complex shadowing effects bring the Ga collection efficiency over 90\% (Fig. 3.a, marker \#80 onwards), the growth is ultimately limited by the amount of Ga impinging the unit cell of the NW array. In our growth conditions, we observe experimentally that the NW maintains a constant axial growth rate, despite an increasing diameter. When combined to a near constant Ga influx, this automatically leads to a decrease of the Ga volume available for the radial growth with the growth duration. If the resulting NWs are not tapered, the radial growth rate $d r / d t$ then diminishes for two reasons: (a) the reduction of Ga volume available for radial growth just mentioned and (b) the increase of nanowire height, which spreads this reducing volume over an increasingly longer height. The reduction of the GaP volume created by radial growth observed between markers \#26-120 thus originates from the mismatch between the increasingly demanding axial growth rate (constant axial elongation, increasing diameter) and the overall Ga collection rate approaching its saturation value.

In conclusion we have precisely measured the height and diameter evolution of selfcatalysed GaP nanowires arranged in an hexagonal array. Over the analysed growth duration (1h), the wire diameter enlarges from $40 \mathrm{~nm}$ to $140 \mathrm{~nm}$ while the wire height grows slightly 
over $3 \mu \mathrm{m}$. In this geometry, significant shadowing effects impact the wire growth to the point that a full analytical treatment is difficult. In addition to the direct shadowing of the NW sidewalls, the surface between each nanowire is shadowed and the re-emitted flux from this surface also suffers from shadowing. Instead we propose a simple numerical scheme which can take into account all shadowing effects in infinite arrays of nanowires. By comparing simulated and experimental fluxes, we draw several conclusions. First, material transfer via surface migration on the mask is unlikely in our growth conditions. Second, over the three different surface re-emitters considered, the simple case of specular reflection gives a better account of the $\mathrm{Ga}$ balance than random uniform or cosine laws, which suggests that Ga atoms simply bounce off the silica mask in our growth conditions.

Supporting information.

- S1 : comparison of several neighbouring NWs

- $\mathrm{S} 2$ : beam equivalent pressure measurements during the NW growth

- S3 : measurement of the radial extension of $\mathrm{Ga}(\mathrm{As}, \mathrm{P})$ markers

- S4 : quantitative chemical analysis of the NW composition

\section{Acknowledgement}

The authors thank Vladimir G. Dubrovskii for inspiring discussions on nanowire growth modelling. The authors acknowledge financial support from French national funding agency ANR under projects NANOCELL (grant no. ANR-15-CE05-0026) and HETONAN (grant no. ANR-15-CE05-0009). The authors also acknowledge ANR "Investissement d'Avenir" program (TEMPOS project no. ANR-10-EQPX-50) for having funded the acquisition of the NANOTEM platform (Dual beam FIB-FEG FEI Scios system and TEM-STEM FEI Titan Themis) used in this work. 


\section{Experimental}

Self-catalyzed GaP NW arrays were grown by molecular beam epitaxy on (111) Si substrates. The hexagonal arrays (500 nm pitch) were defined by electron beam lithography using polymethyl -methacrylate (PMMA) resist on a $20 \mathrm{~nm}$-thick silica layer $\left(\mathrm{SiO}_{2}\right)$ obtained by thermal oxidation. Reactive ion etching $\left(\mathrm{CHF}_{3}+\mathrm{SF}_{6}\right)$ and wet etching ( $\left.\mathrm{HF} 1 \%\right)$ were used to transfer the openings to the silica layer. NW growth was carried out at $600^{\circ} \mathrm{C}$ (as measured by substrate thermocouple), using a Ga flux (Beam equivalent pressure (BEP) $1.8 \times 10^{-7}$ Torr) equivalent to a growth rate $1.35 \AA . \mathrm{s}^{-1}$ on planar (001) GaAs. Phosphorous was provided as $\mathrm{P}_{2}$ using a valved solid cracking cell $\left(\mathrm{BEP} 1.0 \times 10^{-6}\right.$ Torr, cracker temperature $900^{\circ} \mathrm{C}$ ). Arsenic was provided as $\mathrm{As}_{4}$ using a solid valved cracking source (BEP $1.9 \times 10^{-7}$ Torr, cracker temperature $600^{\circ} \mathrm{C}$ ). The growth duration was about 1 hour, starting with $2 \mathrm{~min}$ of Ga deposition without group $\mathrm{V}$ at the growth temperature to form the droplets, followed by 122 repeats of the following growth sequence: GaP for 25 s, Ga(As,P) marker for 5 s. Two specific markers $(\# 41, \# 82)$ are grown for twice as long, $20 \mathrm{~s}$ GaP + 10 s $\mathrm{Ga}(\mathrm{As}, \mathrm{P})$, to provide extra spatial references during transmission electron microscopy observations (see Supplementary Information S1).

After initial observations by scanning electron microscopy (FEI Magellan), the NW arrays were coated by benzocyclobutene $(\mathrm{BCB})$ resist and a slice was extracted by focused ion beam (FEI Scios) to perform transmission electron microscopy (FEI Titan Themis) and detailed chemical analysis (Bruker Super-X).

\section{References}

(1) Jabeen, F.; Grillo, V.; Rubini, S.; Martelli, F. Nanotechnology 2008, 19, 275711.

(2) Colombo, C.; Spirkoska, D.; Frimmer, M.; Abstreiter, G.; Fontcuberta i Morral, A. Phys. Rev. B 2008, 77, 155326. 
(3) Glas, F.; Ramdani, M. R.; Patriarche, G.; Harmand, J.-C. Phys. Rev. B 2013, 88, 195304.

(4) Priante, G.; Ambrosini, S.; Dubrovskii, V. G.; Franciosi, A.; Rubini, S. Cryst. Growth Des. 2013, 13, 3976-3984.

(5) Jacobsson, D.; Panciera, F.; Tersoff, J.; Reuter, M. C.; Lehmann, S.; Hofmann, S.; Dick, K. A.; Ross, F. M. Nature 2016, 531, 317.

(6) Dubrovskii, V. G.; Xu, T.; Díaz Álvarez, A.; Plissard, S. R.; Caroff, P.; Glas, F.; Grandidier, B. Nano Lett. 2015, 15, 5580-5584.

(7) Tersoff, J. Nano Lett. 2015, 15, 6609-6613.

(8) Assali, S.; Zardo, I.; Plissard, S.; Kriegner, D.; Verheijen, M. A.; Bauer, G.; Meijerink, A.; Belabbes, A.; Bechstedt, F.; Haverkort, J. E. M.; Bakkers, E. P. A. M. Nano Lett. 2013, 13, 1559-1563.

(9) Bechstedt, F.; Belabbes, A. J. Phys.: Condens. Matter 2013, 25, 273201.

(10) Greil, J.; Assali, S.; Isono, Y.; Belabbes, A.; Bechstedt, F.; Valega Mackenzie, F. O.; Silov, A. Y.; Bakkers, E. P. A. M.; Haverkort, J. E. M. Nano Lett. 2016, 16, 3703-3709.

(11) Zhang, Y.; Aagesen, M.; Holm, J. V.; Jørgensen, H. I.; Wu, J.; Liu, H. Nano Lett. 2013, 13, 3897-3902.

(12) Priante, G.; Patriarche, G.; Oehler, F.; Glas, F.; Harmand, J.-C. Nano Lett. 2015, 15, 6036-6041.

(13) Madsen, M. H.; Krogstrup, P.; Johnson, E.; Venkatesan, S.; Mühlbauer, E.; Scheu, C.; Sørensen, C. B.; Nygård, J. J. Cryst. Growth 2013, 364, $16-22$.

(14) Harmand, J.-C.; Glas, F.; Patriarche, G. Phys. Rev. B 2010, 81, 235436. 
(15) Ramdani, M. R.; Harmand, J. C.; Glas, F.; Patriarche, G.; Travers, L. Cryst. Growth Des. 2013, 13, 91-96.

(16) Boulanger, J. P.; LaPierre, R. R. J. Cryst. Growth 2014, 388, 116.

(17) Kuyanov, P.; Boulanger, J.; LaPierre, R. R. J. Cryst. Growth 2017, 462, 29-34.

(18) Vukajlovic-Plestina, J.; Kim, W.; Dubrovski, V. G.; Tütüncüoğlu, G.; Lagier, M.; Potts, H.; Friedl, M.; Fontcuberta i Morral, A. Nano Lett. 2017, 17, 4101-4108.

(19) Krogstrup, P.; Madsen, M. H.; Hu, W.; Kozu, M.; Nakata, Y.; Nygård, J.; Takahasi, M.; Feidenhans'l, R. Appl. Phys. Lett. 2012, 100, 093103.

(20) Schroth, P. E. Growth of self-catalyzed GaAs nanowires using molecular-beam-epitaxy and structural characterization by in-situ X-ray diffraction. Ph.D. thesis, Universität Siegen, 2016.

(21) Dalacu, D.; Kam, A.; Austing, D. G.; Wu, X.; Lapointe, J.; Aers, G. C.; Poole, P. J. Nanotechnology 2009, 20, 395602.

(22) Kelrich, A.; Calahorra, Y.; Greenberg, Y.; Gavrilov, A.; Cohen, S.; Ritter, D. Nanotechnology 2013, 24, 475302.

(23) Gibson, S. J.; LaPierre, R. R. Nanotechnology 2014, 25, 415304.

(24) Sabelfeld, K. K.; Kaganer, V. M.; Limbach, F.; Dogan, P.; Brandt, O.; Geelhaar, L.; Riechert, H. Appl. Phys. Lett. 2013, 103, 133105.

(25) Sabelfeld, K.; Kablukova, E. Comput. Mater. Sci. 2016, 125, $284-296$.

(26) Priante, G.; Glas, F.; Patriarche, G.; Pantzas, K.; Oehler, F.; Harmand, J.-C. Nano Lett. 2016, 16, 1917-1924.

(27) Glas, F.; Daudin, B. Phys. Rev. B 2012, 86, 174112. 
(28) Balaghi, L.; Tauchnitz, T.; Hübner, R.; Bischoff, L.; Schneider, H.; Helm, M.; Dimakis, E. Nano Lett. 2016, 16, 4032-4039.

(29) Ruth, V.; Hirth, J. P. J. Chem. Phys. 1964, 41, 3139-3149.

(30) Dubrovskii, V. G.; Cirlin, G. E.; Soshnikov, I. P.; Tonkikh, A. A.; Sibirev, N. V.; Samsonenko, Y. B.; Ustinov, V. M. Phys. Rev. B 2005, 71, 205325.

(31) Krogstrup, P.; Jørgensen, H. I.; Johnson, E.; Madsen, M. H.; Sørensen, C. B.; Fontcuberta i Morral, A.; Aagesen, M.; Nygård, J.; Glas, F. J. Phys. D: Appl. Phys. 2013, 46,313001 .

(32) Krogstrup, P.; Popovitz-Biro, R.; Johnson, E.; Madsen, M. H.; Nygård, J.; Shtrikman, H. Nano Lett. 2010, 10, 4475-4482. 


\section{Graphical TOC Entry}

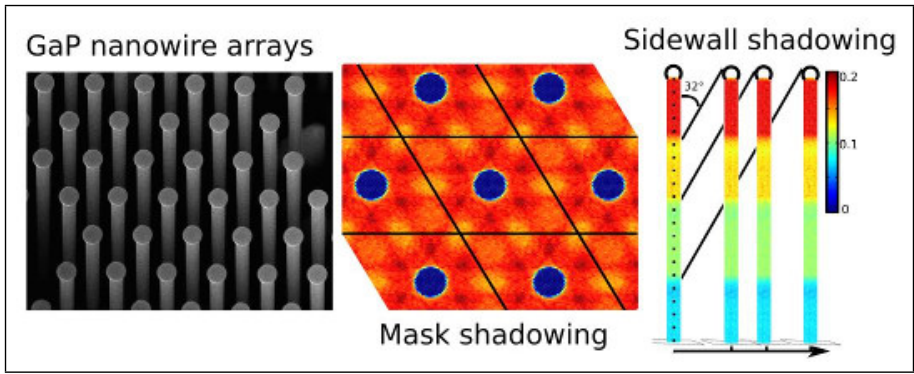

15

16

17

18

19

20

21

22

23

24

25

26

27

28

29

30

31

32

33

34

35

36

37

38

39

40

41

42

43

44

45

46

47

48

49

50

51

52

53

54

55

56

57

58

59

60 